\title{
ESTABLISHING CROP PRODUCTIVITY USING RADARSAT-2
}

\author{
H. McNairn ${ }^{a^{*}}$, J. Shang $^{\text {a }}$, X. Jiao ${ }^{a}$, B. Deschamps ${ }^{b}$ \\ ${ }^{\text {a }}$ Agriculture and Agri-Food Canada, 960 Carling Avenue, Ottawa, Canada K2K 2C2 - heather.mcnairn@agr.gc.ca \\ ${ }^{\mathrm{b}}$ MDA Geospatial Services Inc., 57 Auriga Drive Suite 201, Ottawa, Canada K2E 8B2 - \\ bdeschamps@mdacorporation.com
}

Commission VI, WG VIII/6

KEY WORDS: agriculture, crop, monitoring, SAR, RADARSAT, modelling, retrieval

\begin{abstract}
:
Crop productivity is influenced by a number of management and environmental conditions, and variations in crop growth can occur in-season due to, for example, unfavourable meteorological conditions. Consequently information on crop growth must be temporally frequent in order to adequately characterize crop productivity. Leaf Area Index (LAI) is a key indicator of crop productivity and a number of methods have been developed to derive LAI from optical satellite data. Integration of LAI estimates from synthetic aperture radar (SAR) sensors would assist in efforts to monitor crop production through the growing season, particularly during periods of persistent cloud cover. Consequently, Agriculture and Agri-Food Canada has assessed the capability of RADARSAT-2 data to estimate LAI. The results of a sensitivity analysis revealed that several SAR polarimetric variables were strongly correlated with LAI derived from optical sensors for small grain crops. As the growing season progressed, contributions from volume scattering from the crop canopies increased. This led to the sensitivity of the intensity of linear cross-polarization backscatter, entropy and the Freeman-Durden volume scattering component, to LAI. For wheat and oats, correlations above 0.8 were reported. Following this sensitivity analysis, the Water Cloud Model (WCM) was parameterized using LAI, soil moisture and SAR data. A look up table inversion approach to estimate LAI from SAR parameters, using the WCM, was subsequently developed. This inversion approach can be used to derive LAI from sensors like RADARSAT-2 to support the monitoring of crop condition throughout the cropping season.
\end{abstract}

\section{INTRODUCTION}

Monitoring crop productivity is critical in determining risks to regional and global food security. Gathering the necessary data to monitor productivity is challenging given the acreages involved and the variable nature of crop growth. Crop management applications during active crop growth, as well as ever changing meteorological conditions, mean that crop condition must be monitored continuously through the growing season.

Crop descriptors such as leaf area index (LAI) are good indicators of crop condition and productivity. LAI is the total one-sided green leaf area per unit ground surface area and is a strong indicator of crop production. LAI can be linked with crop yield through process models. Derivation of these crop descriptors from remote sensing data can be used to drive these crop yield models, to validate model estimates and to update or adjust model predictions. Although LAI can be derived from optical sensors (Liu et al., 2010) the reliability of access to data to monitor continuously through the season is questionable due to cloud cover. Synthetic aperture radars (SARs) are thus an appropriate data source to build reliability into monitoring activities. However, the methods to estimate LAI from radar response are not as developed as those that use optical data and thus significant research is required.

To address this knowledge gap, Agriculture and Agri-Food Canada (AAFC) has been investigating the sensitivity of polarimetric SAR data to LAI. This research has included the acquisition of numerous RADARSAT-2 and optical satellite data sets over different cropping regions. In eastern Canada, where corn and soybean production dominates, results have proven the sensitivity of SAR response to LAI for these two broadleaf crops (Jiao et al., 2011). In 2009, the European Space Agency led the AgriSAR campaign under which fully polarimetric RADARSAT-2 data were acquired over three international agriculture research sites. One site was located in western Canada in a region of extensive production of small grains (wheat, oats and barley). The sensitivity of RADARSAT2 to LAI for this class of crops, using the data acquired during AgriSAR, is presented here. The Water Cloud Model (WCM) is then used to model LAI from the radar scattering and a method is proposed to invert the WCM for LAI estimation.

\section{METHODOLOGY}

\subsection{Study Sites and Data Collection}

In 2009, an extensive collection of both optical (RapidEye and Landsat TM) and polarimetric SAR (RADARSAT-2) data were acquired over a site in western Canada. The selected site was AAFC's precision farm located at Indian Head in southern Saskatchewan.

These data were collected under a European Space Agency initiative called AgriSAR. One objective of this campaign was to develop a methodology to estimate crop condition from SAR data. In total, 57 quad-polarimetric ascending and descending RADARSAT-2 images were acquired as part of this AgriSAR initiative. Ground measurements of crop condition, including LAI, were acquired over several small grain (barley, oats,

*Corresponding author 
wheat) fields. Total LAI was measured at 52 sample sites using an LAI-2000 (Li-Cor, Inc., Lincoln, NE) plant canopy analyser under diffuse light conditions. A subset of the satellite data were used in the analysis presented here (Table 1). RADARSAT-2 acquisitions which occurred within one week of optical and ground data collection were selected for further study. One in situ soil moisture station was present in the study site, measuring volumetric soil moisture at a depth of $20 \mathrm{~cm}$.

\begin{tabular}{|l|c|c|c|c|}
\hline $\begin{array}{l}\text { Optical } \\
\text { Data }\end{array}$ & $\begin{array}{c}\text { Optical } \\
\text { Sensor }\end{array}$ & $\begin{array}{c}\text { Field } \\
\text { LAI }\end{array}$ & $\begin{array}{c}\text { RSAT-2 } \\
\text { Data }\end{array}$ & $\begin{array}{c}\text { Beam } \\
\text { Mode }\end{array}$ \\
\hline 2 Jun & RapidEye & 1 Jun & 3 Jun & FQ19 \\
25 Jun & TM & 24 Jun & 24 Jun & FQ14 \\
17 Jul & RapidEye & 15 Jul & & \\
19 Jul & TM & & 21 Jul & FQ19 \\
25 Jul & RapidEye & 24 Jul & 25 Jul & FQ2 \\
10 Aug & RapidEye & & $14 \mathrm{Aug}$ & FQ19 \\
25 Aug & RapidEye & 25 Aug & & \\
\hline
\end{tabular}

Table 1. Data acquired during AgriSAR 2009

\subsection{SAR Data Processing}

The RADARSAT-2 data were processed using PCI Geomatica and the SAR Polarimetry Workstation. Prior to extracting the polarimetric information, a boxcar filter with a 5 by 5 kernel size was applied to the scattering matrix data to suppress SAR speckle. After filtering the covariance matrix was converted to a symmetrized covariance matrix from which intensity backscatter (HH/HV/VV) and intensity ratios (HH/VV, HH/HV, HV/VV) were extracted. Polarimetric variables including total power, pedestal height and complex correlation coefficient (HH-VV) were also extracted from the covariance matrix. Both CloudePottier and Freeman-Durden decompositions were performed on the complex RADARSAT-2 data. Three parameters are derived from the Cloude-Pottier decomposition, namely entropy $(\mathrm{H})$, anisotropy $(\mathrm{A})$, and alpha angle $(\alpha)$. Freeman-Durden decomposition partitions the total power for each image pixel into contributions from three scattering mechanisms: singlebounce, double-bounce, and volume scattering.

Information on the range and azimuth spacing, nadir angle, and satellite altitude for SLC format SAR data were obtained from the SAR production file. Using this information, all the SAR parameters derived above were converted from slant to ground range, followed by an ortho-rectification and geo-referencing procedure using a set of ground control points and national road network vector data.

\subsection{Optical Data Processing}

Atmospheric correction and surface reflectance retrieval of the optical data were accomplished using ATCOR2 implemented in PCI Geomatica. Images were ortho-rectified using platform ephemeris information and models of the internal sensor distortion, ground control points (GCPs) and Digital Elevation Models.

LAI was estimated from the Landsat and RapidEye data using the Modified Triangular Vegetation Index (MTVI2) and a nonlinear curve fitting procedure, as described in Jiao et al. (2011). Strong correlations were found between MTVI2 calculated for near coincident Landsat and RapidEye acquisitions ( $\mathrm{R}^{2}$ of 0.96$)$, as well as between satellite derived LAI and ground measured LAI $\left(\mathrm{R}^{2}\right.$ of 0.78$)$.

An object-based approach was used to compare homogeneous zones of LAI derived from optical data, to SAR response within these homogeneous objects (Jiao et al., 2011). This spatial averaging assists in the reduction of noise inherent in the SAR data. Optical LAI maps were segmented using Definiens software.

\subsection{The Water Cloud Model}

According to Attema and Ulaby (1978), the power backscattered by the whole canopy $\left(\sigma^{0}\right)$ can be represented as the incoherent sum of contributions of the vegetation, $\left(\sigma_{\text {veg }}^{0}\right)$, and the underlying soil, $\left(\sigma_{s o i}^{0}\right)$. The modification of the model by Prevot (1993) was selected here as it incorporates LAI as a descriptor of vegetation development. In this model, SAR backscatter from a canopy at a given incidence angle $(\Theta)$ can be written as:

For the whole canopy:

$$
\sigma^{0}=\sigma_{v e g}^{0}+\tau^{2} \sigma_{\text {soil }}^{0}
$$

where the vegetation contribution is:

$$
\sigma_{\text {veg }}^{0}=A L^{E} \cos \theta\left(1-\tau^{2}\right)
$$

and the soil contribution can be related to the volumetric soil moisture content $\mathrm{M}_{\mathrm{s}}$, expressed in (\%), as:

$$
\sigma_{\text {soil }}^{0}=C+D M_{s}
$$

with

$$
\tau^{2}=\exp (-2 B L / \cos \theta)
$$

where $\tau^{2}$ is the two-way attenuation through the canopy layer, $\mathrm{L}$ is the LAI, expressed in $\left(\mathrm{m}^{2} \mathrm{~m}^{-2}\right)$, the backscatter coefficients $\sigma^{0}, \sigma_{s o i}^{0}$ and $\sigma_{v e g}^{0}$ are expressed in power units. $A, B, C, D$ and $E$ are model coefficients to be defined by experimental data. $A, B$ and $E$ are parameters which depend on canopy type. $E$ is a positive value. Parameters $C$ and $D$ are dependent on soil moisture.

Grouping these terms, the model can be expressed as follows:

$$
\sigma^{0}=A L^{E} \cos \theta(1-\exp (-2 B L / \cos \theta))+\sigma_{\text {soi }}^{0} \exp (-2 B L / \cos \theta)
$$

With only one soil moisture station, too few data were available to parameterize the soil moisture coefficients in the WCM. Consequently the parameterization of $\mathrm{C}$ and $\mathrm{D}$, as determined by Jiao et al. (2011), were used here. Jiao et al. (2011) found that for broadleaf crops, when LAI exceeds 1.0 the SAR response is dominated by the vegetation contributions, with 
only minimally contributions from soil moisture. For example, at a derived LAI of 3.0, 90\% of the total canopy backscatter originates from vegetation contributions at C-band. For the AgriSAR data, the remaining parameters in the model (A, B, and E) were simultaneously determined using a nonlinear least squares method in the Matlab Curve Fitting Toolbox environment, based on the Levenberg-marquardt algorithm.

A look up table (LUT) was produced based on the fitted WCM. LAI values for the look up table ranged from 0 to 8.0 , in increments of 0.01 . Soil moisture ranged from 0 to $50 \%$ in $0.5 \%$ intervals. The LUT was subsequently used to invert the SAR response. The K-nearest neighbour (KNN) search technique was used to find the $\mathrm{K}$ closed points in the LUT to a set of query points (in this case the SAR response). A KD tree was build to facilitate more efficient searching of the LUT.

\section{RESULTS AND DISCUSSION}

\subsection{Sensitivity Analysis}

Correlations between RADARSAT-2 responses and optically derived LAI are presented in Table 2 and Figure 1. As observed for broadleaf crops, SAR parameters which characterize volume scattering from the canopy are most sensitive to grain LAI. These parameters include the linear cross-polarization intensity (HV), entropy and the volume scattering component derived from the Freeman-Durden decomposition. Entropy is calculated by the Cloude-Pottier decomposition and is a measure of the randomness of scattering occurring within a target. As crops emerge and biomass accumulates, the degree of randomness in scattering would be expected to increase.

\begin{tabular}{|l|c|c|c|}
\hline SAR parameter & Wheat & Oats & Barley \\
\hline HH & 0.58 & 0.41 & 0.26 \\
HV & 0.91 & 0.89 & 0.52 \\
VV & 0.26 & -0.28 & -0.46 \\
HV/HH ratio & -0.78 & -0.73 & -0.75 \\
HV/VV ratio & 0.84 & 0.71 & 0.80 \\
HH/VV ratio & 0.69 & 0.75 & 0.80 \\
entropy & 0.94 & 0.90 & 0.81 \\
pedestal height & 0.87 & 0.70 & 0.63 \\
total power & 0.62 & 0.28 & 0.08 \\
volume scattering & 0.86 & 0.89 & 0.39 \\
\hline
\end{tabular}

Table 2. Correlation coefficients (R) between RADARSAT-2 response and optically derived LAI

The correlation between LAI for barley and SAR response was noticeably weaker than that reported for wheat and oats. Significant variation in optical reflectance from these barley fields was observed and may be indicative of greater variability in the growth of this crop.

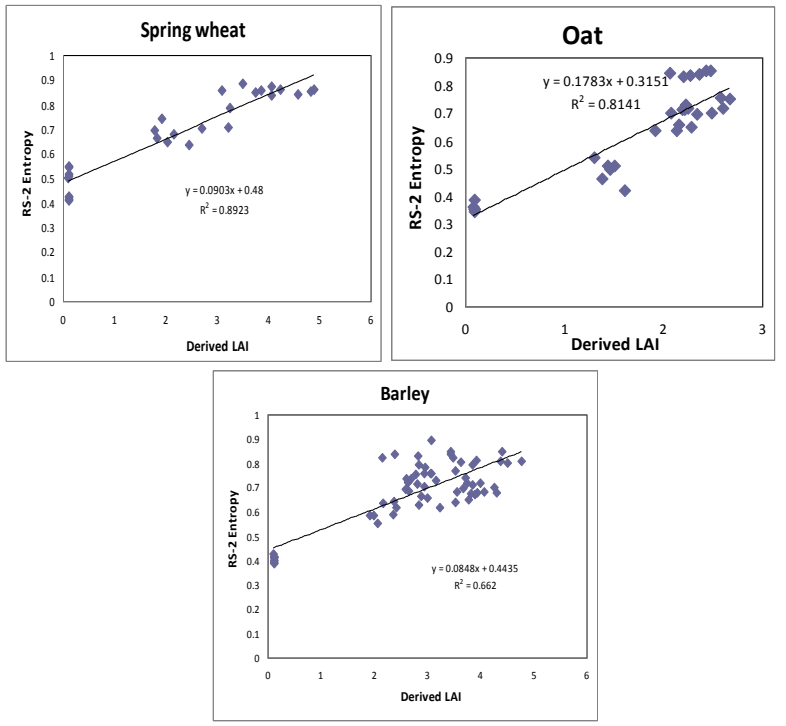

Figure 1. Relationship between entropy and optically derived LAI

There are advantages and disadvantages in selecting either linear intensities or polarimetric variables for LAI estimation. The modelling of LAI from the intensity of backscatter, especially the intensity associated with the cross-polarization, will require a well calibrated sensor. However, many satellite sensors have imaging modes which provide HV backscatter data over wide swaths, necessary for large area monitoring. Provision of parameters from polarimetric decompositions (such as entropy and volume scattering) is restricted to imaging modes of limited swath. RADARSAT-2, for example, provides a wide fine quad-polarimetric mode which acquires data over a swath of only $50 \mathrm{~km}$.

\subsection{Water Cloud Model}

The entropy parameter produced the strongest sensitivity to optically derived LAI. Consequently this parameter was selected for modelling the radar response, and for model inversion. Barley, oats, and wheat were pooled together for the purpose of fitting the WCM, and for model inversion. The degree of model fit is indicated by the coefficient of determination $\left(\mathrm{R}^{2}\right)$. The fit of LAI and soil moisture to entropy, produced a coefficient of determination of 0.7 . The fitted model is displayed in Figure 2. 


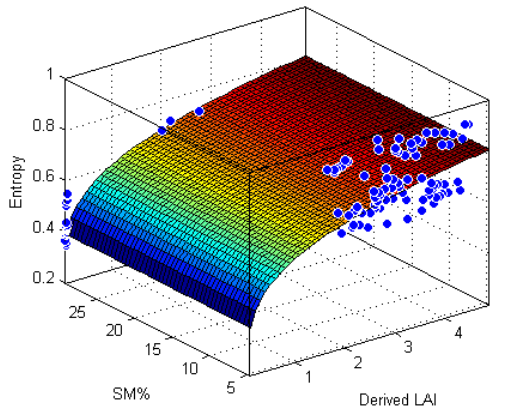

Figure 2. Model fit of LAI and soil moisture to entropy

\subsection{Model Inversion}

The results of the LUT inversion are provided in Figure 3. Here LAI estimates from RADARSAT-2 entropy are compared to LAI derived from the optical data. Some scatter is present but a strong relationship between estimated and derived LAI is present. An underestimation is observed for lower LAI values while the reverse is true at high leaf area.

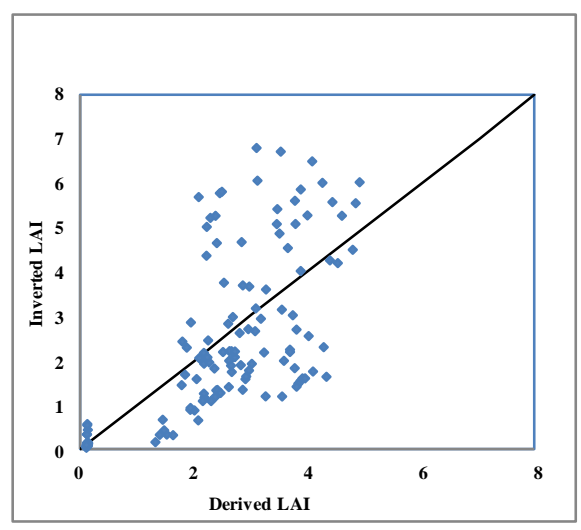

Figure 3. Comparison of LAI inverted from RADARSAT-2 entropy and LAI derived from optical imagery

An LAI map derived from entropy using the Water Cloude Model with the LUT inversion approach is displayed in Figure 4.
RS2 derived LAI map on June 24th

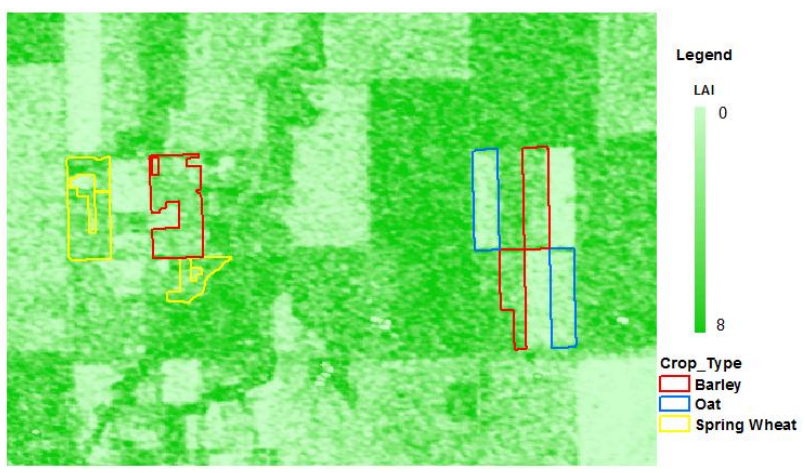

Figure 4. Map of LAI from RADARSAT-2

\section{CONCLUSIONS}

Leaf area index (LAI) is an important parameter for use in monitoring of crop condition and productivity. Although this crop parameter can be derived from optical sensors, reliability in crop monitoring is challenging with these sensors due to cloud cover interference. Thus monitoring activities will require the integration of data from radar sensors.

During the 2009 AgriSAR campaign, RADARSAT-2 polarimetric data were acquired over a site in Canada with extensive small grain production. Results from a sensitivity analysis were consistent with previously published results on corn and soybean LAI. Parameters indicative of characteristics of volume scattering were strongly correlated with LAI for wheat and oats. These included HV intensity backscatter and decomposition parameters derived from the Cloude-Pottier (entropy) and Freeman-Durden (volume scattering) decompositions. Variability in growth conditions for barley led to weaker, although still statistically significant, correlations.

RADARSAT-2 entropy provided the greatest sensitivity to LAI A modified Water Cloud Model (WCM) was subsequently used to model entropy, LAI and soil moisture. A look up table approach was taken to invert the WCM using the entropy response, producing a pixel level map of LAI for the entire RADARSAT-2 image.

These results are consistent with those found with corn and soybean crops (Jiao et al., 2011) and demonstrate the contribution that SAR can provide for monitoring crop condition. Agriculture and Agri-Food Canada has gathered additional data sets and these will be added to the analysis presented here to ensure robustness of this approach to crop monitoring.

\section{REFERENCES}

Attema, E.P.W. and F.T. Ulaby. 1978. Vegetation modelled as a water cloud. Radio Sci., 13, pp. 357-364.

Jiao, X., McNairn, H., Shang, J., Pattey, E., Liu, J., and Champagne, C. 2011. The sensitivity of RADARSAT-2 polarimetric SAR data to corn and soybean Leaf Area Index (LAI), Can. J. Rem. Sens., 37, pp. 69-81. 
International Archives of the Photogrammetry, Remote Sensing and Spatial Information Sciences, Volume XXXIX-B8, 2012 XXII ISPRS Congress, 25 August - 01 September 2012, Melbourne, Australia

Liu, J., Pattey, E., Miller, J.R., McNairn, H., Smith, A., and Hu, B., 2010. Estimating crop stresses, aboveground dry biomass and yield of crops using multi-temporal optical data combined with a radiation use efficiency model. Remote Sens. of Env., 114, pp. 1167-1177.

Prévot, L., Champion, I., and Guyot, G. 1993. Estimating surface soil moisture and leaf area index of a wheat canopy using a dual-frequency ( $\mathrm{C}$ and $\mathrm{X}$ bands) scatterometer. Remote Sens. of Env., 46, pp. 331-339. 